\title{
The hypotensive effect of selective laser trabeculoplasty depending on iridocorneal angle pigmentation in primary open angle glaucoma patients
}

Jaromir T. Wasyluk ${ }^{1}$, Anita Piekarniak-Woźniak², Iwona Grabska-Liberek ${ }^{2}$

\begin{abstract}
1Department of Ophthalmology, Military Institute of Aviation Medicine, Warsaw, Poland ${ }^{2}$ Department of Ophthalmology, Centre of Medical Postgraduate Education, Warsaw, Poland
\end{abstract}

Submitted: 5 September 2012

Accepted: 9 January 2013

Arch Med Sci 2014; 10, 2: 306-308

DOI: 10.5114/aoms.2014.42583

Copyright $\odot 2014$ Termedia \& Banach

\section{Abstract}

Introduction: Selective laser trabeculoplasty (SLT) is applied to reduce intraocular pressure in primary open angle glaucoma therapy. It selectively subjects trabecular pigmented cells to a pulsed, frequency-doubled Nd:YAG laser. The biostimulatory effect on trabecular macrophages was also postulated even in low-pigmented angles. The aim of the study was to assess the hypotensive effectiveness of SLT depending on the degree of iridocorneal angle pigmentation.

Material and methods: Sixty-three eyes of 53 patients with primary open angle glaucoma (POAG) were included in this study and than divided into three subgroups, dependind of iridocorneal angle pigmentation. All subjects underwent SLT on 270 degrees of the angle. Intraocular pressure (IOP) was assesed at baseline and 6 weeks after the laser procedure.

Results: Six weeks post-SLT mean IOP reduction was $2.63 \mathrm{~mm} \mathrm{Hg}$. The degree of IOP reduction showed a significant correlation with angle pigmentation. Intraocular pressure fell by $2.06 \mathrm{~mm} \mathrm{Hg}, 2.46 \mathrm{~mm} \mathrm{Hg}$ and $4.75 \mathrm{~mm} \mathrm{Hg}$ in subgroups with low, marked and high pigmentation, respectively.

Conclusions: The SLT procedure most effectively reduces IOP only in the subgroup of POAG patients with marked angle pigmentation and it is equal to clinically succesfull hypontesive therapeutic effect, according to European Glaucoma Society guidelines. However, lower hypotensive effect in other study subjects may also be the valuable addition to pharmacological therapy of glaucoma.

Key words: selective laser trabeculoplasty, glaucoma, iridocorneal angle.

\section{Introduction}

Primary open angle glaucoma (POAG) is a chronic, progressive optic neuropathy. It involves loss of retinal ganglion cells, resulting in morphological changes of the optic nerve head and specific defects in the visual field (VF).

To date, randomised multi-centre clinical trials, especially EMGT (Early Manifest Glaucoma Trial) [1], have found intraocular pressure (IOP) reduction to be the only effective treatment preventing the emergence of new glaucomatous VF defects. Laser procedures on the iridocorneal angle comprise an approach accepted by the European Glaucoma Society
Corresponding author: Jaromir T. Wasyluk MD, PhD Department of Ophthalmology Military Institute of Aviation Medicine 54/56 Krasińskiego St 01-755 Warsaw, Poland Phone: +48608281286 E-mail: jarowasyluk@gmail.com 
as second line treatment or, alternatively, as first line treatment in selected cases [2].

Selective laser trabeculoplasty (SLT) is a method of non-invasive IOP reduction first reported by Latina [3]. Since then, new indications have been established to include pigmentary and exfoliation glaucoma.

The SLT procedure involves the use of low-energy, Nd:YAG laser second harmonic $(532 \mathrm{~nm}) \mathrm{im}$ pulses applied to pigment-containing cells located in the trabecular meshwork, without causing a sustained coagulation effect.

Until recently, it was thought that the therapeutic (hypotensive) effect of SLT is proportional to the degree of pigmentation of the drainage angle. This was inferred from well-known argon laser trabeculoplasty (ALT), where energy absorption is directly proportional to the level of pigmentation. However, this has not been confirmed for SLT. There have been reports of very good hypotensive effects seen in eyes with paucity or even absence of pigment in the iridocorneal angle. This is possibly attributed to a specific effect of biostimulation, which is postulated to be independent of or marginally dependent on the absorbed dose of energy.

The SLT procedure differs from ALT, not involving coagulation, causing irreversible damage to the trabecular meshwork. The very low energy used in an entire SLT procedure is equal to that used for the single ALT impact.

The aim of the study was to assess of the hypotensive effect of SLT in patients with primary open angle glaucoma, depending on the degree of angle pigmentation.

\section{Material and methods}

The study group comprised 63 eyes of 53 POAG patients (27 females, 26 males), aged 34 to 65 (mean age 50.1). Prior to enrolment, all subjects were on prostaglandin monotherapy, which was maintained throughout the study. Subjects were qualified to have laser surgery either on one eye (43 subjects) or both (10 subjects).

Inclusion criteria: patient informed consent; age 18 to 65; established diagnosis of POAG. Main exclusion criteria: lack of anterior chamber angle visibility on gonioscopy; a history of serious ophthalmologic disease other than glaucoma or any eye surgery; serious systemic disease with a potential to alter visual function.

Subjects underwent a full ophthalmic examination and were divided into subgroups by degree of angle pigmentation, according to the Spaeth grading of Pigment on Trabecular Meshwork (PTM) [4]: a) low pigmentation - PTM +; b) mild pigmentation - PTM ++; c) marked pigmentation - PTM +++.
Baseline IOP was measured at 9.00 AM by Pascal Dynamic Tonometry. Selective laser trabeculoplasty was performed with a Tango ${ }^{\circledR}$ (Ellex) Q-switched Nd:YAG laser. We treated one or both eyes in 43 and 10 cases, respectively. Impacts were applied to $270^{\circ}$ of the angle, i.e. 3 quadrants covered by 100 to 120 spots. The spot size was $400 \mu \mathrm{m}$, pulse duration $3 \mathrm{~ns}$, energy applied 0.5$1.1 \mathrm{~mJ}$, depending on local conditions, to achieve the generation of single gas microbubbles.

The follow-up examination was performed 6 weeks after baseline. The assessment procedure was identical to the baseline evaluation, except for gonioscopy. Tonometry was performed at 9 a.m., as previously.

\section{Results}

The mean baseline IOP for the study group $(n=63)$ was $15.67 \mathrm{~mm} \mathrm{Hg}(\mathrm{SD}=3.51)$. The mean IOP reduction 6 weeks after SLT was statistically significant ( $p<0.001$ ), amounting to $2.63 \mathrm{~mm} \mathrm{Hg}$ (reduction by $16.5 \%, p<0.001, \mathrm{SD}=2.85$ ). Eight eyes (12.6\% of the study group) failed to show significant IOP reduction (Figure 1 ).

The degree of IOP lowering correlated positively and significantly with the degree of iridocorneal angle pigmentation, $p=0.047$ (Kendall Tau b, one-way) (Table I).

\section{Discussion}

Success in glaucoma therapy is usually determined on the basis of an IOP reduction of at least $20 \%$. Such a degree of IOP reduction is hard to achieve with laser and, additionally, the hypo-

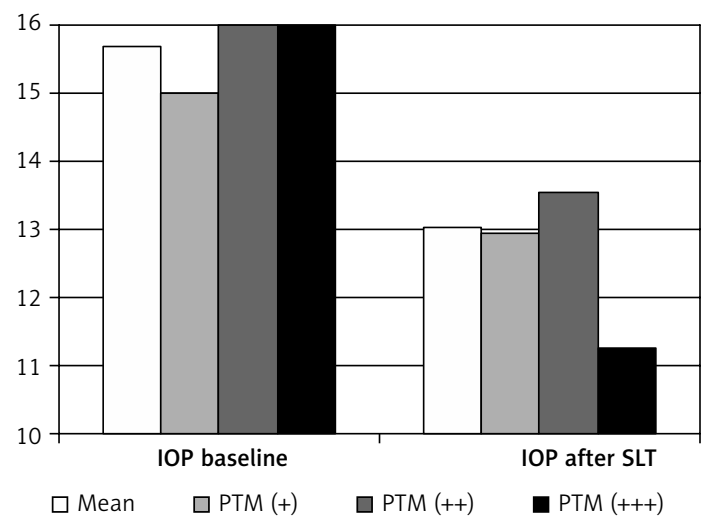

Figure 1. IOP reduction 6 weeks after selective laser trabeculoplasty (in $\mathrm{mm} \mathrm{Hg}$ ) depending on angle pigmentation (PTM)

Table I. Mean IOP reduction in the examined subgroups

\begin{tabular}{|lcc|}
\hline PTM + & $(n=17) 13.7 \%$ & $15.0-12.94 \mathrm{~mm} \mathrm{Hg}$ \\
\hline PTM ++ & $(n=37) 15.3 \%$ & $16.0-13.54 \mathrm{~mm} \mathrm{Hg}$ \\
\hline PTM +++ & $(n=8) 29.6 \%$ & $16.0-11.25 \mathrm{~mm} \mathrm{Hg}$ \\
\hline
\end{tabular}


tensive effect wanes over time, from $81 \%$ of the primary effect after 12 months to only $11 \%$ after 10 years [5]. While these concerns are legitimate in the case of ALT, they do not necessarily apply with SLT, used in this study.

The hypotensive effectiveness of SLT varies, depending on the reporting authors. It ranges from $3.0 \mathrm{~mm} \mathrm{Hg}$ to even $10.6 \mathrm{~mm} \mathrm{Hg}[6,7]$ and is comparable to ALT $[8,9]$. The comparison of outcomes in different reports is difficult. Authors often fail to inform about the portion of the angle circumference treated and the number of burns applied.

The aim of this study was to determine whether the IOP reduction by the non-coagulation SLT procedure depends on the degree of angle pigmentation and if it also effectively works, as postulated before, with low pigmented angles due to the biostimulation effect. It is known that in the case of ALT the effectiveness is linearly determined by pigmentation, amount of energy absorbed and size of coagulation spots.

Our results confirm that SLT effectively lowers mean IOP by week 6 post-surgery. However, the degree of IOP lowering differed significantly in subject subgroups. A clinically satisfactory outcome of $29.6 \%$ ( $4.75 \mathrm{~mm} \mathrm{Hg}$ ) was only achieved in the subgroup with highly pigmented angles.

As said before, some papers suggest that SLT may not be related to degree of angle pigmentation [10] and can also be achieved in patients with minimal presence or even total absence of pigment. Other authors postulate a "biostimulatory" effect, described as the activation and migratory stimulation of phagocytes, as well as conducive metabolic effects exerted by laser on tissues.

In our study, the effect in subjects with lowgrade pigmentation was low or moderate.

However, even the 14-15\% IOP reduction that we achieved seems valuable in view of the results of multicentre trials on glaucoma progression, which suggest that for each $\mathrm{mmHg}$ of IOP reduction the risk of disease progression falls by $10 \%$ [1]. The SLT can be regarded as an adjunct to topical treatment (which was actually the case in our patients). Laser surgery can be considered as an alternative to the addition of another drug (polytherapy).

In conclusion, SLT is an effective treatment method in patients with POAG and marked iridocorneal angle pigmentation; we may assume that laser surgery can be sufficiently effective, even when applied as a first-line, single modality in such patients. Also less pigmented eyes can benefit from IOP-lowering to some extent, which makes it a viable addition to the topical therapy. One may assume that this treatment could be correspondingly effective in other glaucoma types, where the angle is also open and the degree of pigmentation high, as in pigmentary or exfoliation glaucoma.
References

1. Heijl A, Leske MC, Bengtsson B, Bengtsson B, Hussein M, Early Manifest Glaucoma Trial Group. Measuring visual field progression in the Early Manifest Glaucoma Trial. Acta Ophthalmol Scand 2003; 81: 286-93.

2. European Glaucoma Society. Terminology and guidelines for glaucoma. 3rd Edition. Editrice Dogma, Savona, Italy 2008; 151-2.

3. Latina MA, Park C. Selective targeting of trabecular meshwork cells: in vitro studies of pulsed and CW laser interactions. Exp Eye Res 1995; 60: 359-71.

4. Spaeth GL. The normal development of the human anterior chamber angle: a new system of descriptive grading. Trans Ophthalmol Soc UK 1971; 91: 709-39.

5. Moulin F, Haut J. Argon laser trabeculoplasty. Results over 10 years. J Fr Ophthalmol1994; 17: 93-8.

6. Melamed S, Ben Simon GJ, Levkovitch-Verbin H. Selective laser trabeculoplasty as primary treatment for open-angle glaucoma: a prospective, nonrandomized pilot study. Arch Ophthalmol 2003; 121: 957-60.

7. Lanzetta P, Menchini U, Virgili G. Immediate intraocular pressure response to selective laser trabeculoplasty. $\mathrm{Br}$ J Ophthalmol 1999; 83: 29-32.

8. Martinez-de-la-Casa JM, Garcia-Feijoo J, Castillo A, et al. Selective vs argon laser trabeculoplasty: hypotensive efficacy, anterior chamber inflammation, and postoperative pain. Eye 2004; 18: 498-50.

9. Damji KF, Bovell AM, Hodge WG, et al. Selective laser trabeculoplasty versus argon laser trabeculoplasty: results from a 1-year randomised clinical trial. Br J Ophthalmol 2006; 90: 1490-4.

10. Hodge WG, Damji KF, Rock W, Buhrmann R, Bovell AM, Pan Y. Baseline IOP predicts selective laser trabeculoplasty success at 1 year post-treatment: results from a randomised clinical trial. Br J Ophthalmol 2005; 89: 1157-60. 